\section{Assistência e mortalidade neonatal no setor público do Município do Rio de Janeiro, Brasil: uma análise do período 1994/2000}

\author{
Neonatal care and mortality in public hospitals \\ in Rio de Janeiro, Brazil, 1994/2000
}

\author{
1 Instituto Fernandes \\ Figueira, Fundação Oswaldo \\ Cruz, Rio de Janeiro, Brasil. \\ 2 Secretaria Municipal \\ de Saúde do Rio de Janeiro, \\ Rio de Janeiro, Brasil. \\ Correspondência \\ M. A. S. M. Gomes \\ Instituto Fernandes Figueira, \\ Fundação Oswaldo Cruz. \\ Rua Eurico Cruz 39, \\ apto. 101, Rio de Janeiro, $R J$ \\ 22461-200, Brasil. \\ maria@iff.fiocruz.br
}

\begin{abstract}
This article analyzes an intervention by the Rio de Janeiro Municipal Health Department (SMS$R J)$, Brazil, to reduce the neonatal mortality rate (strategies for organizing and upgrading neonatal care in the municipal system, including an increase in the number of neonatal high-risk beds). We studied the trends in neonatal mortality rate (1995/2000), neonatal care provided in different public hospitals (1994/2000), and admissions profile and mortality in four neonatal intensive care units (NICUs) under the SMS-RJ (2000). There was a concentration of high-risk neonatal care in the municipal hospitals (an increase from 28.0\% of the care provided for live premature neonates in 1994 to $67.0 \%$ in 2000) and a reduction in the neonatal mortality rate in units under the Unified National Health System (from 19.9 deaths per thousand live births in 1996 to 15.5 in 2000). There was no reduction in the prematurity and low birth weight rates among mothers residing in the municipality of Rio de Janeiro. Analysis of admissions to the NICUs showed a high proportion of neonates born to mothers from municipalities outside Rio de Janeiro, while $14.0 \%$ of the mothers had not received prenatal care, and the mortality rate among newborns with birth weight $<1.500 \mathrm{~g}$ was $32.0 \%$.
\end{abstract}

Services Evaluation; Infant Mortality; Neonatal Intensive Care Units
Maria Auxiliadora de Souza Mendes Gomes 1,2 José Maria Andrade Lopes 1 Maria Elizabeth Lopes Moreira 1 Nicole Oliveira Mota Gianini 1,2

\section{Introdução}

A redução do componente pós-neonatal do coeficiente de mortalidade infantil no Brasil 1, observada ao longo das décadas de 80 e 90, contribuiu para a maior visibilidade da mortalidade neonatal e para a crescente produção de estudos sobre mortalidade neonatal em nosso meio 2,3,4,5,6,7,8,9,10.

Embora o maior peso do componente neonatal no coeficiente de mortalidade infantil seja um padrão identificado na transição epidemiológica de outros países, encontramos especificidades em nosso meio, uma vez que a inversão entre os componentes neonatal e pósneonatal, observada nos dados nacionais desde meados da década de 90, não foi acompanhada pela redução da mortalidade neonatal evitável 11,12.

Encontramos, num contexto desfavorável de dificuldades de acesso, iniqüidade e precariedade da assistência perinatal, elementos que potencialmente explicam a estabilidade ou mesmo o aumento no coeficiente de mortalidade neonatal no país 13. Entretanto, outros elementos devem ser considerados. Dentre eles, destacamos fatores que têm contribuído, nos últimos anos, para a redução do sub-registro dos óbitos neonatais, como, por exemplo, a urbanização, o incremento de cobertura de notificação de óbitos e a maior informação sobre assistência ao parto em unidades de saúde 14 . 
Da mesma forma, assim como observado em outros países, não podemos desconsiderar a influência das mudanças na percepção dos limites de viabilidade nos registros da mortalidade neonatal, levando à inclusão de óbitos anteriormente classificados dentro da natimortalidade 15,16 .

No Município do Rio de Janeiro, Brasil, no início da década de 90, a avaliação sobre a capacidade instalada na área obstétrica evidenciava importante deficiência resultante da diminuição e/ou instabilidade na oferta de leitos disponíveis: mais de $50,0 \%$ dos leitos federais e estaduais encontravam-se desativados 17 . No tocante ao atendimento neonatal de maior complexidade (leitos de Unidade Intermediária e de Terapia Intensiva), as dificuldades eram ainda mais acentuadas. Além do déficit existente na capacidade instalada, a carência quase absoluta desse tipo de atendimento nos demais municípios da região metropolitana e no restante do Estado 18 era responsável por acentuada migração de pacientes de risco para a capital 19 .

Além do diagnóstico de carência de leitos, estudos sobre aspectos da qualidade da assistência perinatal realizados na capital e região metropolitana do Rio de Janeiro confirmavam as dificuldades de acesso e a má qualidade da (des)atenção prestada: descaso com o acompanhamento pré-natal; falta de continuidade entre o pré-natal e o atendimento ao parto; falhas grosseiras na condução do trabalho de parto, parto e do atendimento ao neonato; dificuldades para internação durante trabalho de parto; e intercorrências evitáveis no atendimento neonatal estão retratados nos trabalhos de Carvalho 2, Gomes 19 e Nogueira 20.

A partir de 1994, são implementadas, no âmbito da gestão municipal do Rio de Janeiro, uma série de estratégias visando à ampliação, qualificação e humanização da assistência obstétrica e neonatal nas unidades da Secretaria Municipal de Saúde (SMS-RJ), com o objetivo de reduzir a mortalidade materna e neonatal 21. Neste artigo, apresentamos uma análise do atendimento neonatal no setor público do $\mathrm{Mu}$ nicípio do Rio de Janeiro, com base em indicadores sobre nascimentos e óbitos neonatais na rede pública própria ou conveniada ao Sistema Único de Saúde (SUS), referentes ao período 1994/2000, até chegar a um ângulo mais fechado de observação: características e mortalidade dos neonatos internados em quatro Unidades de Terapia Intensiva Neonatal da SMS-RJ, considerando que a utilização de indicadores mais próximos da prática assistencial pode contribuir para melhor entendimento da assistên- cia neonatal no setor público do Município do Rio de Janeiro.

\section{Método}

O delineamento metodológico deste estudo foi definido no campo das investigações avaliativas 22,23 e utilizou o modelo de análise de intervenção, avaliando a capacidade dos recursos que foram mobilizados e dos serviços que foram produzidos (estratégias de ampliação, humanização e qualificação da assistência obstétrica e neonatal na rede municipal) para atingir um dos objetivos definidos (redução da mortalidade neonatal).

Definimos como intervenção o conjunto de estratégias implementadas pelo gestor municipal composto pelos seguintes itens: estabelecimento de sistema de informação na área perinatal pela implantação e gerenciamento por meio da SMS-RJ das bases de dados Sistema de Informação sobre Nascidos Vivos (SINASC) e Sistema de Informação sobre Mortalidade (SIM), municipalização de quatro maternidades federais em agosto de 1995 e uma em janeiro de 2000, municipalização de Postos de Assistência Médica (PAM), investimentos na estrutura das maternidades sob gestão municipal (obras e aquisição de equipamentos para ampliação ou readequação da capacidade instalada de leitos obstétricos e neonatais, ampliação dos contratos dos serviços de apoio e manutenção dos equipamentos e lotação de recursos humanos). Esse processo resultou no aumento da oferta de consultas pré-natais, no número de maternidades (de 4 para 9) e de leitos sob gestão municipal, passando de 535 para 567 leitos obstétricos e de 135 para 234 leitos neonatais. Os leitos neonatais da rede municipal passaram a representar cerca de 50,0\% dos leitos existentes no setor público do Município $(\mathrm{n}=463)$ do Rio de Janeiro 18.

Ainda como componente da intervenção, encontramos a implementação dos Conselhos Técnicos de Neonatologia e de Obstetrícia, integrados pelas chefias médica e de enfermagem das maternidades municipais. Aos conselhos técnicos, coordenados pela equipe central, coube a responsabilidade da operacionalização de um amplo leque de requisitos necessários à qualificação do atendimento perinatal: incorporação tecnológica, padronização de rotinas e protocolos clínicos para atendimento ambulatorial e hospitalar, capacitação dos profissionais, entre outros 24 .

Como indicadores dos resultados da intervenção, além da análise da variação do coefi- 
ciente de mortalidade neonatal dos nascimentos ocorridos nas instituições públicas e conveniadas ao SUS, ao final do período estudado, utilizamos: variação na taxa de prematuridade (idade gestacional < 37 semanas) e taxa de baixo peso ao nascer (peso $<2.500$ g) entre mães residentes no Município do Rio de Janeiro (optamos, neste caso, por utilizar o dado para mães residentes, uma vez que, a utilização dos dados referentes aos nascimentos ocorridos poderia espelhar apenas a migração de pacientes de risco para a capital), distribuição global e segundo perfil de risco (caracterizado por idade gestacional < 37 semanas - pré-termo e peso ao nascer $<1.500 \mathrm{~g}-$ muito baixo peso ao nascer) dos nascimentos ocorridos em unidades públicas ou conveniadas ao SUS, no Município do Rio de Janeiro, segundo tipo de prestador e a variação na taxa de muito baixo peso ao nascer, segundo município de residência da mãe e tipo de prestador. Incluímos ainda a análise do perfil das internações neonatais em quatro Unidades de Terapia Intensiva Neonatal da SMS-RJ.

A análise das taxas de mortalidade, prematuridade, baixo peso ao nascer e do perfil dos nascidos vivos nos diferentes prestadores (municipais, estaduais, federais, universitários e conveniados) foi feita por intermédio dos bancos de dados SIM e SINASC para o período 1996/ 2000 disponibilizados pela Gerência de Informações Epidemiológicas da Coordenação de Epidemiologia da SMS-RJ. Não foi possível analisar as taxas de mortalidade para os anos de 1994 e 1995 por problemas operacionais na base de dados que não possibilitaram a discriminação dos óbitos por prestador.

Para o estudo do perfil do atendimento neonatal na SMS-RJ, foram selecionadas as quatro maternidades de maior porte, com o maior número de leitos neonatais (26-53 leitos) e com maior proporção de nascidos vivos prematuros em 1999 (14,6\%-31,2\%). Foram analisados os prontuários de todos os recém-nascidos internados nas quatro Unidades de Terapia Intensiva Neonatal $(\mathrm{n}=565)$ selecionadas para o estudo, durante o período de 27 de maio de 2000 a 27 de setembro de 2000. Os dados das unidades neonatais da SMS-RJ foram comparados com os dados de unidades neonatais canadenses e sul-americanas 25,26 . Os dados foram coletados dos prontuários e digitados e analisados com utilização do programa Epi Info (versão 6.04), do Centers for Disease Control and Prevention (CDC).

\section{Resultados e discussão}

\author{
Indicadores perinatais e atendimento \\ neonatal no setor público do Município \\ do Rio de Janeiro
}

Na análise do banco de dados SINASC, no período 1994/2000, não encontramos uma redução significativa nas taxas de prematuridade e de baixo peso ao nascer, entre neonatos de mães residentes no Município do Rio de Janeiro, acompanhando a intervenção executada pelo gestor municipal. A taxa de baixo peso ao nascer, que era de 9,5\% em 1994, manteve-se em 9,1\% em 2000. Mais preocupante, entretanto, foi o aumento observado na taxa de prematuridade: de 6,1\% em 1994 para 7,8\% em 2000.

No tocante ao papel dos diferentes prestadores na assistência ao parto, as informações do SINASC evidenciam a crescente participação das unidades municipais na assistência perinatal prestada no âmbito do SUS no Município do Rio de Janeiro: de 17,0\% dos 68.351 nascidos vivos no SUS em 1994 para $47,0 \%$ dos 72.456 nascidos vivos em 2000. Com o intuito de aprofundar a análise da prestação do cuidado perinatal no Município do Rio de Janeiro, apresentamos, na Tabela 1, a participação dos diferentes prestadores na assistência neonatal de risco. Para tanto, utilizaremos como indicadores a distribuição dos nascimentos prematuros e com peso ao nascer menor que $1.500 \mathrm{~g}$ (muito baixo peso ao nascer) ocorridos na rede pública ou conveniada ao SUS ao longo da segunda metade da década de 90 .

$\mathrm{Na}$ análise da Tabela 1, observamos que a maior participação da rede municipal na assistência perinatal foi acompanhada também por uma maior participação na assistência do grupo de risco: cerca de $67,0 \%$ dos nascimentos prematuros e $60,0 \%$ dos com muito baixo peso ocorridos em 2000, na rede pública do município, ocorreram em unidades da SMS-RJ. Lembramos que essa participação na assistência neonatal de risco é maior do que a proporção da rede municipal em termos de capacidade instalada de leitos neonatais no setor público do Município do Rio de Janeiro que, conforme mencionado previamente, representa cerca de $50,0 \%$.

No período 1994/2000, encontramos uma redução significativa na participação das unidades sob gestão federal na assistência perinatal de risco, em função do processo de municipalização das maternidades federais. Todavia, é importante registrar que a soma das parcelas de atendimento de nascidos vivos prematuros das unidades federais $(31,83 \%)$ e municipais 
Tabela 1

Distribuição percentual dos nascimentos prematuros (PT) e muito baixo peso ao nascer (MBPN) ocorridos no SUS por prestador.

Município do Rio de Janeiro, Brasil, 1994/2000.

\begin{tabular}{|c|c|c|c|c|c|c|c|c|}
\hline \multirow[t]{2}{*}{ Prestador } & \multicolumn{2}{|c|}{$1994(n=68.351)$} & \multicolumn{2}{|c|}{$1996(n=68.949)$} & \multicolumn{2}{|c|}{$1998(n=70.146)$} & \multicolumn{2}{|c|}{$2000(n=72.456)$} \\
\hline & $\begin{array}{c}\text { PT } \\
(n=5.057)\end{array}$ & $\begin{array}{c}\text { MBPN } \\
(n=1.219)\end{array}$ & $\begin{array}{c}\mathrm{PT} \\
(\mathrm{n}=6.212)\end{array}$ & $\begin{array}{c}\text { MBPB } \\
(n=1.336)\end{array}$ & $\begin{array}{c}P T \\
(n=6.573)\end{array}$ & $\begin{array}{c}\text { MBPN } \\
(n=1.454)\end{array}$ & $\begin{array}{c}\text { PT } \\
(n=7.239)\end{array}$ & $\begin{array}{c}\text { MBPN } \\
(n=1.545)\end{array}$ \\
\hline Secretaria Municipal de Saúde (\%) & 27,97 & 30,77 & 60,94 & 61,67 & 67,49 & 64,10 & 66,61 & 60,40 \\
\hline Secretaria Estadual de Saúde (\%) & 5,87 & 9,59 & 8,01 & 8,60 & 10,64 & 12,04 & 11,37 & 12,29 \\
\hline Ministério da Saúde (\%) & 31,83 & 41,34 & 14,17 & 15,05 & 14,49 & 16,23 & 15,08 & 19,54 \\
\hline Universitário (\%) & 7,85 & 6,80 & 7,30 & 7,86 & 4,37 & 5,15 & 4,67 & 5,83 \\
\hline Conveniado + filantrópico (\%) & 26,48 & 11,50 & 9,58 & 6,82 & 3,01 & 2,48 & 2,27 & 1,94 \\
\hline Total (\%) & 100,00 & 100,00 & 100,00 & 100,00 & 100,00 & 100,00 & 100,00 & 100,00 \\
\hline
\end{tabular}

Fonte: Sistema de Informação sobre Nascidos Vivos/Gerência de Informações Epidemiológicas, Coordenação de Epidemiologia,

Superintendência de Saúde Coletiva, Secretaria Municipal de Saúde do Rio de Janeiro.

$(27,97 \%)$ no ano de 1994 , comparada com a mesma soma em $2000(15,08 \%$ e $66,61 \%$, respectivamente), mostra um aumento de $59,8 \%$ para $81,7 \%$. O aumento observado demonstra, portanto, que a maior participação da SMS-RJ no atendimento perinatal de risco não ocorreu somente em função da simples transferência da parcela "federal" do atendimento pela municipalização dessas unidades.

Deve ser ressaltada a expressiva variação negativa observada na participação da rede conveniada ao SUS (unidades privadas e filantrópicas) no atendimento dos nascimentos prematuros, passando de $26,48 \%$ em 1994 para $2,27 \%$ dos nascimentos ocorridos em 2000. Encontramos esse mesmo perfil quando analisamos a participação da rede conveniada no atendimento de nascidos vivos com muito baixo peso ao nascer. Dentro da perspectiva da hierarquização dos serviços, ressaltamos como um elemento bastante positivo a progressiva redução na participação das unidades conveniadas ao SUS no atendimento de nascidos vivos prematuros (de $26,48 \%$ em 1994 para 2,27\% em 2000) ou com muito baixo peso ao nascer (de 11,5\% em 1994 para 1,94\% em 2000). A menor participação dos prestadores conveniados no atendimento de neonatos prematuros e com muito baixo peso reflete uma seleção "desejável” na clientela atendida nesses serviços, uma vez que os mesmos não estão credenciados para o atendimento de maior complexidade.

As unidades universitárias também apresentaram, no período, redução na participação no atendimento de neonatos prematuros no Município do Rio de Janeiro: de 7,85\% em 1994 para $4,67 \%$ em 2000 . A pequena participação desses serviços no atendimento perinatal de risco foi uma outra característica importante encontra- da no Município do Rio de Janeiro, o que se contrapõe à lógica observada nos países desenvolvidos de organização de grandes centros terciários ligados a serviços universitários 25 .

Em relação ao perfil de risco segundo residência da mãe, a análise da tendência do muito baixo peso ao nascer, evidencia sua maior ocorrência entre mães não residentes no Município do Rio de Janeiro, característica que se acentuou no período do estudo. Entre 1994 e 2000, a proporção de nascimentos de bebês com peso $<1.500 \mathrm{~g}$ entre mães residentes no Município do Rio de Janeiro passou de 1,6\% para 2,34\%. No grupo de nascidos vivos de mães moradoras de outros municípios, esse índice passou de 3,45\% para 6,04\%. Em 2000, dos 1.545 nascimentos com peso menor que $1.500 \mathrm{~g}$, ocorridos no SUS no Município do Rio de Janeiro, 468 (30,3\%) foram neonatos de mães moradoras de outros municípios. As unidades municipais foram as principais responsáveis por esse atendimento $(55,1 \%)$, seguidas pelas unidades federais $(28,4 \%)$. Esse dado reforça o reconhecimento do perfil de risco das gestantes oriundas de outros municípios que buscam atendimento em unidades do Município do Rio de Janeiro, em função da carência desse atendimento nos demais municípios do Estado.

\section{Mortalidade neonatal dos nascimentos ocorridos no setor público do Município do Rio de Janeiro}

Antes de apresentarmos a variação na taxa de mortalidade neonatal entre os nascidos no setor público do Município do Rio de Janeiro, julgamos importante discutir alguns aspectos do coeficiente de mortalidade neonatal neste $\mathrm{Mu}$ nicípio. A tendência histórica dos componen- 
tes da mortalidade infantil, no Município do Rio de Janeiro (décadas de 80 e 90), acompanhou o perfil, já descrito, de redução da mortalidade basicamente em função da redução do componente pós-neonatal 4,7 , confirmando um padrão nacional de tendência desse indicador 1 .

Contudo, a partir da segunda metade da década de 90, podemos verificar uma tendência de queda na mortalidade neonatal, contrastando com a tendência de estabilidade ou mesmo elevação nesse coeficiente observada no período anterior: 12,8/1.000 nascidos vivos em 1990, 15,2/1.000 em 1994 e 11,2/1.000 em 200024.

Ainda no que tange à situação da mortalidade neonatal no Município do Rio de Janeiro, apresentamos, na Tabela 2, a evolução da mortalidade neonatal por faixas de peso selecionadas. A análise dessas taxas, no período 1996/ 2000, mostra que a tendência de redução da mortalidade neonatal global espelha uma variação negativa também no grupo de menor peso, merecendo destaque a variação negativa de $33,8 \%$ no grupo entre 1.000 e $1.500 \mathrm{~g}$.

Tendo em vista o objetivo de analisar, mais especificamente, a variação na mortalidade neonatal no setor público, apresentamos na Figura 1, a taxa de mortalidade neonatal dos nascimentos ocorridos no Município do Rio de Janeiro, segundo tipo de prestador: rede pública ou conveniada ao SUS e rede privada, no período 1996/2000, já que, conforme já relatado na descrição do Método, não foi possível obter a discriminação por prestador nos dados referentes aos anos de 1994 e 1995.

A comparação da taxa de mortalidade neonatal entre nascidos em instituições do SUS e da rede privada confirma a distância desses indicadores, com menores taxas entre os nascidos na rede privada; porém, sem variação negativa no período estudado. No grupo de nascidos no setor público, encontramos uma variação negativa de $22,0 \%$, passando de 19,9 óbitos por mil nascidos vivos em 1996 para 15,5 em 2000.

Por esses indicadores, identificamos, também para a mortalidade neonatal entre os nascimentos ocorridos no setor público, uma tendência de queda que acompanha a tendência apontada acima para este indicador para os residentes no Município do Rio de Janeiro. Reconhecendo que, no período estudado, a mortalidade neonatal dos nascidos vivos na rede privada já era de fato menor e que este mesmo perfil se repete entre os nascimentos de mães residentes no Município do Rio de Janeiro, podemos discutir que a redução na taxa de mortalidade entre os nascimentos ocorridos no setor público contribuiu para a redução do coeficiente do Município. Neste ponto, considera-
Tabela 2

Percentual de mortalidade neonatal proporcional em faixas de peso selecionadas. Município do Rio de Janeiro, Brasil, 1996/2000.

\begin{tabular}{lccc}
\hline & $<1.000 \mathrm{~g}(\%)$ & $\mathbf{1 . 0 0 0 - 1 . 4 9 9} \mathbf{g}(\%)$ & $\mathbf{1 . 5 0 0 - 2 . 4 9 9 g ( \% )}$ \\
\hline 1996 & 77,7 & 32,1 & 4,1 \\
1998 & 71,5 & 26,4 & 3,3 \\
2000 & 57,4 & 21,3 & 2,9 \\
Variação 1996/2000 & $-26,1$ & $-33,8$ & $-28,3$ \\
\hline
\end{tabular}

Fonte: Sistema de Informação sobre Mortalidade/Sistema de Informação sobre Nascidos Vivos/Gerência de Informações Epidemiológicas, Coordenação de Epidemiologia, Superintendência de Saúde Coletiva, Secretaria Municipal de Saúde do Rio de Janeiro.

Figura 1

Taxas de mortalidade neonatal dos nascimentos ocorridos no Município do Rio de Janeiro, Brasil, segundo prestador, 1995/2000.

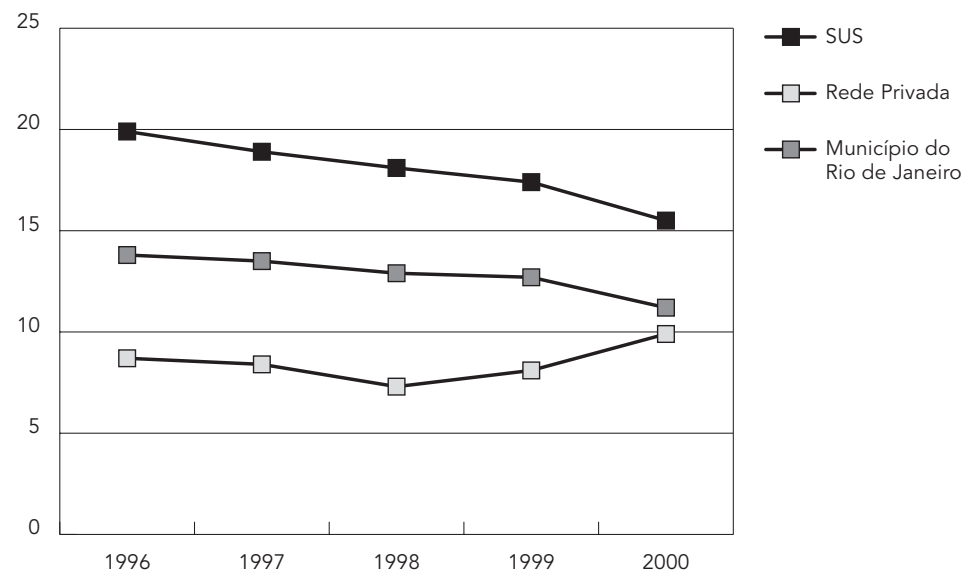

Fonte: Gerência de Informações Epidemiológicas, Coordenação de Epidemiologia, Superintendência de Saúde Coletiva, Secretaria Municipal de Saúde do Rio de Janeiro.

mos importante destacar que observamos uma variação negativa na taxa de mortalidade dos nascimentos ocorridos nas instituições do SUS, apesar da crescente migração de pacientes de alto risco residentes em outros municípios apontada anteriormente.

Perfil do atendimento em quatro Unidades de Terapia Intensiva Neonatal da Secretaria Municipal de Saúde do Rio de Janeiro (SMS-RJ)

Durante o período de coleta de dados, a taxa média de ocupação nessas unidades variou en- 
tre 100,0-125,0\%, confirmando um padrão de "superlotação" que, certamente, prejudica a adequação da assistência prestada e contribui para maior risco de morbi-mortalidade neonatal 24. Na avaliação das características maternas dos neonatos internados, encontramos proporção considerável de internações de recém-natos de mães moradoras de outros municípios. No estrato com peso ao nascer $<1.500 \mathrm{~g}$, esse grupo representou 33,1\% das internações. Em relação à faixa etária, encontramos uma média de 25,4 anos (13-46 anos), com 13,9\% de mães que não realizaram nenhuma consulta pré-natal e $18,3 \%$ com tempo de bolsa rota maior que 24 horas. Os diagnósticos maternos mencionados com maior freqüência foram: hipertensão (24,2\%), trabalho de parto prematuro (11,8\%), amniorrexe prematura (11,3\%), oligodramnia (6,9\%), infecção urinária $(6,2 \%)$, sofrimento fetal $(5,5 \%)$ e descolamento prematuro de placenta $(3,5 \%)$.

$\mathrm{Na}$ Tabela 3, apresentamos algumas das características da população internada nas unidades municipais no período do estudo, comparando com o perfil da população descrita em um estudo colaborativo canadense - Canadian NICU Network, incluindo informações a respeito de 20.488 admissões em 17 unidades neonatais, no período de janeiro de 1996 a outubro de 199725.

Encontramos, nas unidades estudadas, uma proporção de $95,5 \%$ de neonatos que nasceram nos próprios serviços, confirmando o perfil de concentração do risco perinatal a partir do atendimento obstétrico. No estudo canadense, essa proporção foi menor: do total de neonatos atendidos, cerca de $26,0 \%$ vieram de outras unidades. Embora, a princípio, esse fato pudesse apontar para uma situação mais adequada nas unidades cariocas (na perspectiva de

Tabela 3

Características das internações neonatais em unidades da Secretaria Municipal de Saúde do Rio de Janeiro (SMS-RJ) e Canadian NICU Network.

\begin{tabular}{lcc}
\hline Características & SMS-RJ & $\begin{array}{c}\text { Canadian NICU } \\
\text { Network }\end{array}$ \\
\hline BPN (\%) & 71,0 & 53,0 \\
MBPN (\%) & 30,0 & 20,0 \\
$<37$ semanas (\%) & 81,0 & 65,0 \\
Sem pré-natal (\%) & 14,0 & 2,0 \\
Tempo médio de internação (dias) & 38 & 19 \\
Transferências de outras unidades (\%) & 4,5 & 26,0 \\
\hline
\end{tabular}

BPN = baixo peso ao nascer; $\mathrm{MBPN}=$ muito baixo peso ao nascer. minimizar os riscos do transporte neonatal), deve ser mencionado que isso não reflete uma organização racional e hierarquizada do atendimento. Nas unidades públicas do Município do Rio de Janeiro, as gestantes internadas em situação de risco chegam, em geral, pelos meios próprios, muitas vezes em trabalho de parto ou em outras situações de urgências obstétricas 19.

$\mathrm{Na}$ análise dos prontuários, outro fato que confirma a falta de avaliação e referência de risco é a pequena proporção, nas unidades estudadas, de recém-natos cujas mães tenham feito pré-natal em maternidades de referência para atendimento de risco: cerca de $21,0 \%$.

Por fim, merece ser destacada a diferença entre a não-realização de pré-natal entre as mães dos dois estudos. Encontramos, nas unidades da SMS-RJ, cerca de $14,0 \%$ de neonatos cujas mães não tiveram acesso ao cuidado prénatal, valor sete vezes maior do que o relatado nas unidades canadenses $(2,0 \%)$.

No que diz respeito ao peso ao nascer, encontramos uma proporção mais elevada de baixo peso ao nascer e muito baixo peso ao nascer nas internações das unidades do estudo quando comparado às unidades do Canadá, apontando para um perfil de maior risco da população internada nas unidades da SMS-RJ.

Na comparação do tempo médio de internação, encontramos nas unidades da rede municipal um período total de internação médio de 38 dias na unidade neonatal (Unidade de Terapia Intensiva mais Unidade Intermediária). Esse período é bem maior que o observado nas unidades canadenses, que apresentaram uma média de internação de 19 dias. Nesta comparação, deve ser ressaltada uma característica das altas no Canadá: apenas $47,0 \%$ dos bebês tiveram alta diretamente para o domicílio. Os outros $43,0 \%$ receberam alta da unidade terciária sendo referenciados para hospitais de menor complexidade, fato que pode explicar, parcialmente, esse menor tempo médio de internação. Além desse fato, não podemos desconsiderar outros fatores da dinâmica de funcionamento das unidades neonatais em estudo que podem, potencialmente, prolongar o período de internação em função de complicações causadas por falhas na estrutura e no processo de atendimento.

Em relação à sobrevida após internação neonatal, encontramos as unidades da SMS-RJ numa situação bem mais desfavorável. A taxa de mortalidade entre as internações com peso menor que $1.500 \mathrm{~g}$ foi mais que o dobro da observada nas unidades canadenses: $32,0 \%$ contra $13,0 \%$. A comparação com as unidades neo- 
natais sul-americanas 26 coloca as unidades da SMS-RJ numa situação mais próxima: a mortalidade no grupo com muito baixo peso ao nascer nesses serviços foi de $27,0 \%$ (Tabela 4 ).

Ainda que a mortalidade no grupo com muito baixo peso ao nascer seja um indicador que deva ser melhorado (segundo demonstrado na comparação com o índice canadense), é importante ressaltar que a análise da mortalidade entre neonatos com muito baixo peso deve ser feita também em dois estratos: $<1.000 \mathrm{~g}$ e 1.000-1.500g, visto que existem diferenças importantes de morbi-mortalidade e de prognóstico entre esses dois grupos 27. Encontramos, no período de coleta de dados, nessas quatro unidades, taxas de mortalidade que variaram entre $62,5 \%$ e $82,3 \%$ entre neonatos com peso $<1.000$ g e $13,3 \%$ e $16,0 \%$ no grupo com peso 1.000-1.500g. Lembramos que, de acordo com o apresentado na Tabela 2, para o Município como um todo, em 2000, a mortalidade nesse segundo grupo de peso foi de $21,3 \%$. Por fim, cabe ressaltar a importância dos quadros infecciosos como causa de mortalidade nas unidades neonatais estudadas: a infecção foi mencionada como causa em cerca de $40,0 \%$ do total de óbitos.

\section{Considerações finais}

A análise da participação dos diferentes prestadores na assistência perinatal mostra que a intervenção implementada pelo gestor municipal mudou o panorama da atenção perinatal no setor público do Município do Rio de Janeiro, concentrando o atendimento perinatal de risco na rede municipal e contribuindo para o alcance do objetivo de redução da mortalidade neonatal entre os nascimentos ocorridos nas instituições do SUS do Município do Rio de Janeiro. Por outro lado, a intervenção realizada não foi suficiente para a redução nas taxas de prematuridade e de baixo peso ao nascer, entre as mães residentes no Município do Rio de Ja- neiro. Por mais que a redução nessas taxas não tenha sido definida diretamente como objetivo da intervenção desenvolvida, entendemos que, em função da bem estabelecida correlação desses fatores com a ocorrência do óbito neonatal, a capacidade da intervenção na redução da mortalidade neonatal seria fortalecida com a inclusão de estratégias que efetivamente reduzissem a ocorrência desses eventos.

Logo, os desafios ainda são muitos. As taxas de prematuridade e de baixo peso ao nascer e a mortalidade neonatal dos nascimentos ocorridos nas instituições do SUS do Município do Rio de Janeiro permanecem altos, se comparados a outros países 10. A análise por um ângulo mais fechado de observação, a comparação do perfil de atendimento e da mortalidade no grupo com muito baixo peso ao nascer entre as unidades da SMS-RJ e as da Canadian NICU Network, cumpriu seu objetivo de melhor delineamento da assistência neonatal e evidenciou, ainda mais, a magnitude desses desafios. Neste contexto, algumas ações estratégicas devem ser incansavelmente perseguidas. Dentre elas, destacamos as que garantam a estrutura necessária ao funcionamento das unidades neonatais, a adequação do processo de atendimento (incluindo a "erradicação" definitiva da "superlotação") e o monitoramento sistemático das práticas assistenciais e dos resultados neonatais.

Com base nas informações obtidas por intermédio deste estudo, enfatizamos ainda que os demais "elos" da cadeia de eventos perinatais (assistência pré-natal e ao parto) devem ser objeto de crescente intervenção. A estabilidade na taxa de baixo peso ao nascer e o aumento na taxa de prematuridade (mesmo levando-se em conta possíveis interferências da já mencionada mudança na percepção dos limites de viabilidade), os diagnósticos maternos e a elevada proporção de neonatos internados em Unidades de Terapia Intensiva Neonatal cujas mães não fizeram pré-natal são indícios suficientes de que a perspectiva do cui-

Mortalidade neonatal no grupo com muito baixo peso ao nascer (MBPN) entre as unidades da Secretaria Municipal de Saúde do Rio de Janeiro (SMS-RJ), Canadian NICU Network e Grupo Colaborativo Neocosur.

\begin{tabular}{lccc}
\hline & SMS-RJ & $\begin{array}{c}\text { Canadian NICU } \\
\text { Network }\end{array}$ & $\begin{array}{c}\text { Grupo Colaborativo } \\
\text { Neocosur }\end{array}$ \\
\hline Mortalidade (\%) & 32,0 & 13,0 & 27,0 \\
Variação entre as unidades (\%) & $28,0-37,0$ & - & $11,0-51,0$ \\
\hline
\end{tabular}


dado perinatal precisa ser incorporada de fato, tanto na formulação das políticas e programas de saúde da mulher e da criança quanto na prática assistencial. Não foi de outra forma que países com melhores resultados perinatais atingiram seus objetivos 28,29,30.

Nesta mesma perspectiva, a organização e qualificação da assistência perinatal em todo o contexto da região metropolitana e interior do Estado são pontos de intervenção urgente (a expressiva migração sem a responsabilização ou referência formal dos sistemas locais de saúde de gestantes de risco de outros municípios para a capital é uma prova contundente dessa premissa). O enfrentamento desses pontos críticos passa necessariamente pela hierarquização e regionalização dos diferentes serviços a partir de uma coordenação integrada e articulada dos diferentes gestores responsáveis pela atenção perinatal no Rio de Janeiro.

\section{Resumo}

Este artigo apresenta uma avaliação da intervenção realizada pela Secretaria Municipal de Saúde do Rio de Janeiro(SMS-RJ) (estratégias de organização e qualificação da assistência neonatal na rede municipal, incluindo a ampliação dos leitos neonatais de risco), com o objetivo de reduzir a mortalidade neonatal. Analisamos as mudanças ocorridas no atendimento dos diferentes prestadores do setor público (período 1994/2000), na taxa de mortalidade neonatal dos nascimentos ocorridos nas instituições do Sistema Único de Saúde (1995/2000) e o perfil das internações em quatro Unidades de Terapia Intensiva Neonatal (UTIN) da SMS-RJ (2000). Encontramos a concentração do atendimento neonatal de risco nas unidades municipais (de 28,0\% do atendimento de nascidos vivos prematuros, em 1994, para 67,0\% em 2000), redução na mortalidade neonatal dos nascimentos ocorridos no SUS (de 19,9 óbitos por mil nascidos vivos em 1996 para 15,5 em 2000). Não houve redução nas taxas de prematuridade e baixo peso ao nascer entre as mães residentes no Município do Rio de Janeiro. Na análise das internações nas UTIN encontramos elevada proporção de neonatos de mães moradoras de outros municípios, $14,0 \%$ de mães que não realizaram pré-natale $32,0 \%$ de mortalidade entre neonatos com peso ao nascer $<1.500 \mathrm{~g}$.

Avaliação dos Serviços; Mortalidade Infantil; Unidades de Terapia Intensiva Neonatal

\section{Colaboradores}

M. A. S. M. Gomes foi responsável pelo desenho metodológico, trabalho de campo, análise das bases de dados e discussão dos resultados. J. M. A. Lopes participou da definição do desenho metodológico e da discussão dos resultados. M. E. L. Moreira contribuiu na análise e na discussão dos resultados. N. O. M. Gianini colaborou na discussão dos resultados. 


\section{Referências}

1. Simões CC. Estimativas da mortalidade infantil por microrregiões e municípios. Brasília: Secretaria de Políticas de Saúde, Ministério da Saúde; 1999.

2. Carvalho ML. Mortalidade neonatal e aspectos da qualidade da atenção à saúde na região metropolitana do Rio de Janeiro em 1986/87 [Dissertação de Mestrado]. Rio de Janeiro: Escola Nacional de Saúde Pública, Fundação Oswaldo Cruz; 1993.

3. Almeida NMGS. Mortalidade perinatal no Município de São Paulo [Dissertação de Mestrado]. São Paulo: Faculdade de Saúde Pública, Universidade de São Paulo; 1996.

4. Leal MC, Scwarcwald CL. Evolução da mortalidade neonatal no Estado do Rio de Janeiro, Brasil (1979-1993): análise por causa segundo grupo de idade e região de residência. Cad Saúde Pública 1996; 12:243-52.

5. Gomes JO, Santo AH. Mortalidade infantil em município da região Centro-Oeste Paulista, Brasil, 1990 a 1992. Rev Saúde Pública 1997; 31:330-41.

6. Araújo BF, Bozzetti MC. Mortalidade neonatal precoce no Município de Caxias do Sul: um estudo de coorte. J Pediatr (Rio J) 2000; 76:200-6.

7. Campos TP, Carvalho MS, Barcellos CC. Mortalidade infantil no Rio de Janeiro, Brasil: áreas de risco e trajetória dos pacientes até os serviços de saúde. Rev Panam Salud Pública 2000; 8:164-71.

8. Ribeiro VS, Silva AAM. Tendências da mortalidade neonatal em São Luís, Maranhão, Brasil, de 1976 a 1996. Cad Saúde Pública 2000; 16:429-38.

9. Magalhães MC. Atenção hospitalar perinatal e a mortalidade neonatal no Município de Juiz de Fora [Dissertação de Mestrado]. Rio de Janeiro: Escola Nacional de Saúde Pública, Fundação Oswaldo Cruz; 2000.

10. Lansky S, França E, Leal MC. Mortalidade perinatal e evitabilidade: revisão de literatura. Rev Saúde Pública 2002; 36:759-72.

11. Ortiz-Flores LP. Características da mortalidade neonatal no Estado de São Paulo [Tese de Doutorado]. São Paulo: Faculdade de Saúde Pública, Universidade de São Paulo; 1999.

12. Lansky S, França E, Leal MC. Mortes perinatais evitáveis em Belo Horizonte, Minas Gerais, Brasil, 1999. Cad de Saúde Pública 2002; 18:1389-400.

13. Ministério da Saúde. A mortalidade perinatal e neonatal no Brasil. Brasília: Ministério da Saúde; 1998.

14. Lannes R. Responsabilidade partilhada: o papel das instâncias do SUS na organização da assistência perinatal. Tema (Proj. RADIS) 1999; (17):2-5.

15. Howell EM, Blondel B. International infant mortality rates: bias from reporting differences. Am J Public Health 1994; 84:850-2.

16. Joseph KS, Allen A, Kramer MS, Cyr M, Fair M. Changes in the registration of stillbirths $<500 \mathrm{~g}$ in Canada, 1985-1995. Paediatr Perinat Epidemiol 1999; 13:278-87.

17. Israel G, Vellozo V. Programa de Assistência Integral à Saúde da Mulher (PAISM): uma abordagem qualitativa de experiências desenvolvidas no âmbito das Secretarias Municipais de Saúde. Rio de Janeiro: Instituto Brasileiro de Administração $\mathrm{Mu}$ nicipal; 1994.
18. Conselho Regional de Medicina do Estado do Rio de Janeiro. Assistência materno - infantil no Estado do Rio de Janeiro. Rio de Janeiro: Conselho Regional de Medicina do Estado do Rio de Janeiro; 1998.

19. Gomes MASM. Aspectos da qualidade do atendimento à gestação e ao parto através da percepção das usuárias [Dissertação de Mestrado]. Rio de Janeiro: Instituto Fernandes Figueira, Fundação Oswaldo Cruz; 1995.

20. Nogueira MI. Assistência pré-natal em Duque de Caxias: um caso de violência institucional [Dissertação de Mestrado]. Rio de Janeiro: Escola Nacional de Saúde Pública, Fundação Oswaldo Cruz; 1993.

21. Valladares DP. Ações de contracepção e assistência ao parto: a experiência do Rio de Janeiro In: Giffin K, Costa SH, organizadores. Questões de saúde reprodutiva. Rio de Janeiro: Editora Fiocruz; 1999. p. 357-76.

22. Contandriopoulus AP, Champagne F, Potvin L, Denis JL, Boyle P. Saber preparar uma pesquisa: definição, estrutura, financiamento. São Paulo: Editora Hucitec/Rio de Janeiro: ABRASCO; 1994.

23. Hartz ZMA, organizador. Avaliação em saúde: dos modelos conceituais à prática na análise da implantação de programas. Rio de Janeiro: Editora Fiocruz; 1997.

24. Gomes MASM. Assistência neonatal na Secretaria Municipal de Saúde do Rio de Janeiro: uma análise do período 1995-2000 [Tese de Doutorado]. Rio de Janeiro: Instituto Fernandes Figueira, Fundação Oswaldo Cruz; 2002.

25. Lee SKM, McMillan DD, Ohlsson A, Pendray M, Synnes A, Whyte R, et al. Variations in practice and outcomes in the Canadian NICU Network: 1996-1997. Pediatrics 2000; 106:1070-9.

26. Tapia JL. Very-low-birth weight infant outcomes in 11 South American NICUs. J Perinatol 2002; 22:2-7.

27. Hack M, Horbar JD, Malloy MH, Tyson JE, Wright EW, Wright L. Very low birth weight outcomes of National Institute of Child Health and Human Development Neonatal Network. Pediatrics 1991; 87:587-97.

28. Riegel K. Determinants for special neonatal care in Perinatal Care Delivery Systems - description and evaluation in European community countries. Comission of the European Communities Health Services Research Series 1. England: Oxford Medical Publications; 1986.

29. Saugstad OD. Perinatal health in Europe: neonatal aspects. In: Carrera JM, Cabero L, Baraibar R, editors. The perinatal medicine of the new millenium. Barcelona: Monduzzi Editore; 2001. p. 1-4.

30. Papiernick E. Regionalisation of perinatal care in Europe. In: Carrera JM, Cabero L, Baraibar R, editors. The perinatal medicine of the new millenium. Barcelona: Monduzzi Editore; 2001. p. 1295-7.

Recebido em 02/Fev/2004

Versão final reapresentada em 08/Ago/2004

Aprovado em 21/Fev/2005 